\title{
Tratamento de exposição a estímulos e treinamento de habilidades como coadjuvantes no manejo do craving em um dependente de crack
}

\author{
Cue exposure treatment and coping skills training as adjuvant therapies \\ in the management of craving in a crack cocaine addic
}

\begin{abstract}
Renata Brasil Araujo ${ }^{1}$, Alexandre Didó Balbinot ${ }^{2}$, Maria da Graça Tanori de Castro3 ${ }^{3}$ Marcelo Rossoni da Rocha ${ }^{4}$
\end{abstract} Sandro Rene Pinto de Sousa Miguel ${ }^{5}$, Mírian Cohen ${ }^{5}$, Rosemeri Siqueira Pedroso ${ }^{6}$

\begin{abstract}
Resumo
Objetivo: Tem-se observado um aumento da prevalência de dependentes de crack em amostras clínicas, o que torna necessária a realização de pesquisas quanto a estratégias de tratamento direcionadas a essa clientela. $O$ objetivo deste estudo foi descrever o caso de um dependente de crack internado no qual foram utilizados o tratamento de exposição a estímulos (TEE) e o treinamento de habilidades ( $\mathrm{TH}$ ) como coadjuvantes ao tratamento tradicional.

Descrição do caso: O paciente é do sexo masculino, 29 anos de idade, solteiro, ensino médio completo. Era dependente de crack e de maconha e fazia uso nocivo de álcool. O paciente já estava internado havia 2 semanas e tinha passado por um protocolo de quatro sessões com entrevista motivacional e prevenção à recaída. Foram feitas seis sessões, ao longo de 2 semanas, de TEE e TH, nas quais o paciente foi exposto in vivo e pela imaginação a estímulos evocadores de fissura, como cachimbo de crack, isqueiro, pedras simuladas, lembranças de locais e amigos associados ao uso da droga. Ele também foi treinado para utilizar estratégias de manejo da fissura. Após 3 meses da alta hospitalar, foi realizado screening toxicológico para avaliar a manutenção de abstinência. O paciente avaliou o uso das técnicas como importante para a manutenção da abstinência após 3 meses da alta e para sua baixa média de fissura pelo crack.

Comentários: Talvez o TEE e o TH para manejo da fissura possam ser úteis como coadjuvantes no tratamento de dependentes de crack. Tal uso deve ser avaliado em ensaios clínicos para demonstrar seu real benefício.
\end{abstract}

Descritores: Usuários de drogas, cocaína crack, transtornos relacionados ao uso de substâncias.

\begin{abstract}
Objective: An increased prevalence of crack cocaine users has been observed in clinical samples over the past years, underscoring the need for conducting research and developing treatment strategies aimed at this population. The objective of this study was to describe the case of a crack cocaine addict (inpatient) submitted to cue exposure treatment (CET) and coping skills training (CS) as adjuvant approaches to the conventional addiction treatment.
\end{abstract}

Case description: A male patient, 29 years old, single, with complete high school, was dependent on crack cocaine and marijuana and also had a diagnosis of harmful alcohol use. The patient had been hospitalized for 2 weeks and had undertaken a protocol comprising four motivational interview and relapse prevention sessions. Six sessions of CET and CS were carried out over 2 weeks, including both in vivo and imaginal exposure to stimuli evoking craving for crack cocaine, such as a crack pipe, a lighter, and simulated crack cocaine rocks (in vivo exposure), or remembering places and friends associated with drug use (imaginal exposure). The patient was also trained to use strategies for the management of craving. Three months after hospital discharge, toxicological screening was performed to assess abstinence. CET and CS were considered by the patient to be important techniques for the maintenance of abstinence and of a low level of craving 3 months after discharge.

Comments: These findings suggest that the two techniques may be useful as adjuvant therapies in the treatment of crack cocaine dependents. CET and CS should be assessed in clinical trials in order to demonstrate their real benefits.

Keywords: Drug users, crack cocaine, substance-related disorders.

\footnotetext{
${ }_{1}^{1}$ Doutora em Psicologia, Pontifícia Universidade Católica do Rio Grande do Sul (PUCRS), Porto Alegre, RS. Coordenadora, Programa de Dependência Química, Hospital Psiquiátrico São Pedro, Porto Alegre, RS. ${ }^{2}$ Residente, Educação Física, Hospital Psiquiátrico São Pedro. ${ }^{3}$ Psicóloga. Mestre em Psicologia Clínica, PUCRS. ${ }^{4}$ Acadêmico de Psicologia Clínica, PUCRS. Estagiário, Psicologia Clínica, Hospital Psiquiátrico São Pedro. ${ }^{5}$ Residente, Psiquiatria, Hospital Psiquiátrico São Pedro. ${ }^{6}$ Doutoranda, Ciências Médicas: Psiquiatria, UFRGS.

Recebido em 12/10/2010. Aceito em 22/12/2010. Não foram declarados conflitos de interesse associados à publicação deste artigo.

Como citar: Araujo RB, Balbinot AD, Castro MGT, Rocha MR, Miguel SRPS, Cohen M, et al. Tratamento de exposição a estímulos e treinamento de habilidades como coadjuvantes no manejo do craving em um dependente de crack. Trends Psychiatry Psychother. 2011;33(3):181-8.
} 


\section{Introdução}

A fissura - ou craving - associada ao uso de cra$c k$ é um fenômeno que vem sendo discutido mais frequentemente devido ao aumento da prevalência do uso dessa substância em amostras clínicas ${ }^{1}$. Como o pico de ação do crack é muito rápido (cerca de 8 a 10 segundos após o consumo) e seu efeito é fugaz, os usuários em pouco tempo relatam desejo de usar a droga novamen$t^{2}$. Não existem medicações comprovadamente eficazes para tratar a fissura da cocaína/crack; no entanto, vários estudos estão sendo feitos com vários fármacos, como o valproato ${ }^{3}$, que não mostrou bons resultados. Outras classes de fármacos avaliadas, com resultados pouco promissores, foram os antidepressivos ${ }^{4}$ e a gabapentina5. O topiramato apresentou resultados mais promissores $^{6}$, bem como $\mathrm{N}$-acetilcisteína7, modafinili ${ }^{8}$, dissulfiram $^{7,9}$, ondansetron ${ }^{8}$ e a imunoterapia ${ }^{8}$.

Nos transtornos de adição, tanto a fissura quanto a recaída podem ocorrer mesmo após longos períodos de abstinência, tornando seu tratamento um desafio para os profissionais que trabalham nessa área ${ }^{10}$. Os estímulos associados ao uso de drogas evocam a memória da experiência desse uso ${ }^{11}$ e apresentam propriedades reforçadoras que promovem a repetição do comportamento. Especificamente com relação à cocaína, a liberação de dopamina no corpo estriado aumenta quando dependentes dessa substância olham um estímulo associado a seu consumo ${ }^{12}$.

O estudo do papel das respostas condicionadas na recaída motivaram o início do estudo das técnicas de exposição a estímulos no tratamento de dependentes químicos. Entre essas respostas condicionadas encontram-se alterações na frequência cardíaca, na condutância da pele e no relato de fissura ${ }^{13,14}$. Foltin \& Haney ${ }^{13}$ demonstraram que as respostas condicionadas podem ser reduzidas após várias sessões de extinção.

O tratamento de exposição a estímulos (TEE) é utilizado como coadjuvante no atendimento de dependentes de substâncias psicoativas como álcool ${ }^{15}$, ópio ${ }^{16}$, nicotina ${ }^{17}$ e cocaína ${ }^{18}$. Segundo Childress et al. ${ }^{19}$, a apresentação continuada de estímulos condicionados a um dependente, sem que este possa fazer uso da substância, gera uma diminuição das respostas condicionadas fisiológicas e de fissura através da extinção. Os autores estudaram 37 dependentes de cocaína, com período de abstinência entre 7 e 10 dias, randomizados em quatro grupos, como segue: 1) psicoterapia de suporte + extinção; 2) psicoterapia de suporte + atividades para compensar a atenção voltada aos pacientes que participavam do grupo que faria a extinção; 3) aconselhamento em adição + extinção; e 4) aconselhamento em adição + atividades para compensar a atenção voltada aos pacientes que participavam do grupo que faria a extinção. Todas as modalidades de tratamento duravam 2 semanas em regime hospitalar. Na segunda fase do estudo, houve seguimento ambulatorial por 2 meses, com sessões semanais de extinção ou atividades substitutas e psicoterapia ou aconselhamento, conforme o grupo. Os resultados mostraram uma redução da fissura e dos sintomas que mimetizavam o efeito ou os sintomas da abstinência. A redução foi mais gradual na fissura e mais rápida nas outras duas variáveis subjetivas avaliadas, sendo que a extinção quase total foi alcançada após a sexta sessão. No que diz respeito às variáveis fisiológicas, houve diminuição tanto da temperatura corporal quando da condutância da pele durante as sessões de exposição, mas mesmo após a última sessão, essas variáveis ainda apresentavam alterações. No período de seguimento de 2 meses, os dois grupos tratados com as técnicas de exposição apresentaram maior retenção no tratamento e menor uso de cocaína controlado por screening de urina. No entanto, esses sujeitos relataram continuar apresentando elevada fissura associada a estímulos que não puderam ser simulados no regime hospitalar. Dessa forma, os autores concluíram que a generalização da extinção aos estímulos não é completa no "mundo real"19. É importante salientar que a resposta condicionada só ocorria nos sujeitos dependentes de cocaína quando estes eram expostos a estímulos específicos. Por outro lado, o aumento da fissura era menos frequentemente acompanhado por sintomas similares àqueles causados pela substância propriamente dita, tais como sensação de $h i g h^{19}$.

Deve-se destacar que existem alguns vieses na avaliação do TEE, como o fato de esse tipo de tratamento ter sido planejado para ser apenas um coadjuvante no tratamento de dependentes químicos ${ }^{20}$. Assim, devem sempre ser oferecidas outras modalidades terapêuticas associadas, como a entrevista motivacional ${ }^{21}$, a terapia cognitivo-comportamental ${ }^{22}$ e a prevenção à recaída ${ }^{23}$. Essa necessidade de combinação do TEE com outras terapias atrapalha a avaliação de seus resultados.

Embora existam vários estudos relacionados ao TEE com dependentes químicos, não há nenhum estudo disponível realizado com dependentes de crack. Assim, o objetivo deste estudo foi descrever o caso de um dependente de crack no qual foi utilizado o TEE associado ao treinamento de habilidades $(\mathrm{TH})$ para o manejo da fissura ${ }^{15}$, como coadjuvantes ao tratamento tradicional.

\section{Descrição do caso}

Este estudo descreve o caso de um sujeito do sexo masculino, separado, com 26 anos de idade, ensino médio completo, que residia com os pais e tinha um filho que vivia com sua ex-companheira. Estava hospitalizado 
na Unidade de Dependência Química Jurandy Barcellos do Hospital Psiquiátrico São Pedro, em Porto Alegre (RS). Apresentava diagnóstico de dependência de cocaína/crack e maconha e uso nocivo de álcool de acordo com a Classificação Estatística de Doenças e Problemas Relacionados à Saúde - $10^{a}$ Revisão (CID-10) ${ }^{24}$. O crack era sua droga favorita. Iniciou o uso de maconha, cocaína aspirada e álcool aos 15 anos, e de crack aos 24. Antes da internação, usava uma média semanal de 80 pedras de crack, 1 cigarro de maconha e 1 garrafa de cerveja ( $750 \mathrm{ml}$ ). Deixou de usar cocaína aspirada quando iniciou o uso do crack. Estava abstinente de todas as substâncias psicoativas havia 21 dias no momento do início das atividades de TEE e TH.

Antes do tratamento, o paciente realizou quatro entrevistas individuais, sendo uma para anamnese e aplicação do Structured Clinical Interview for DSM Disorders $\left(\right.$ SCID-IV-TR) ${ }^{25}$, duas para entrevista motivacional ${ }^{22} \mathrm{e}$ uma para prevenção da recaída ${ }^{23}$. Além disso, estava internado havia 2 semanas, com o objetivo de interromper o uso de todas as substâncias psicoativas, e disposto a permanecer por mais 2 semanas internado - critérios necessários para a inclusão no TEE e no TH. Não apresentava sintomas psicóticos, déficits cognitivos e/ou atencionais que prejudicassem seu desempenho na intervenção. Na entrevista diagnóstica através do SCID-IV-TR ${ }^{25}$, administrada por residente de psiquiatria devidamente treinado, não foram identificadas comorbidades psiquiátricas. Deve-se destacar que o paciente apresentava-se motivado não só para interromper o uso de substâncias psicoativas como também para fazer o tratamento desde o primeiro dia de internação. Sua história de vida poderia ser resumida, desde o início do uso de drogas, como uma sucessão de períodos longos passados em fazendas de recuperação (cerca de 9 meses) e períodos curtos morando com os pais nos quais recaía, sendo considerado necessário, para ele e para a família, o retorno ao tratamento em fazenda. Na unidade, sempre foi discreto, escolhendo se relacionar com pacientes com um perfil de mais responsabilidade, o que ele relatava como sendo o seu "modo de ser". Apresentava funcionamento rígido e com alto nível de exigência para consigo mesmo e para com os demais, com traços de personalidade anancástica ${ }^{24}$.

A escolha deste sujeito para o estudo foi feita pela profissional que adaptou o protocolo, por ter este sido o primeiro paciente internado que preencheu os critérios de inclusão para o TEE e o TH após a adaptação do protocolo e a realização de um estudo piloto envolvendo cinco sujeitos.

\section{Instrumentos}

Os seguintes instrumentos foram utilizados no diagnóstico e tratamento do paciente:
1) Entrevista estruturada, com o objetivo de definir o perfil do paciente, avaliar seu consumo de crack e de outras substâncias psicoativas, bem como sua motivação para interromper o uso do crack.

2) Inventário de Avaliação para o Tratamento de Exposição aos Gatilhos para o Uso do Crack (IATEGCRACK), questionário que avalia o uso do crack com relação a materiais usados, situações de risco e estratégias de enfrentamento (Araujo RB, 2009, material não publicado).

3) Cartão de Fissura e Confiança, adaptado de Monti et al. $^{20}$, para dependentes de crack. Neste cartão, o paciente usa escalas analógicas visuais para atribuir notas de 0 a 10 à fissura que está sentindo no momento e ao grau de confiança que ele tem de que não usaria o crack se de fato essa substância estivesse na sua frente.

4) Registro da Fissura e Confiança - Material do Terapeuta, em que o terapeuta anota os graus de fissura e confiança marcados pelo paciente, o tempo, a frequência cardíaca, a ferramenta que o paciente utilizou para o manejo da fissura e a nota de 0 a 10 que ele deu para esta última ( 0 = nada eficiente; $10=$ muito eficiente).

5) Materiais para exposição in vivo, ou seja, parafernália do uso do crack (cachimbos, isqueiros, cinzas de cigarro, pedras de crack fantasia), elaborados de acordo com a descrição obtida via IATEGCRACK.

6) Frequencímetro para verificar a frequência cardíaca do paciente em cada mudança nos níveis de fissura e confiança.

7) Cronômetro para marcar o tempo de cada mudança nas pontuações de fissura e confiança.

8) Questionário de Ferramentas Pessoais de Manejo de Fissura pelo Crack, adaptado de Monti et al. ${ }^{20}$, que inclui estratégias de enfrentamento para o manejo da fissura, notas dadas pelo paciente nas sessões e tipos de imagens mentais que mais funcionaram para diminuir a fissura.

9) Questionário para avaliar o consumo de substâncias, situações de risco e estratégias de enfrentamento pós-alta, aplicado 3 meses após a alta do paciente.

10) Screening toxicológico de urina, para avaliar o consumo de substâncias psicoativas.

\section{Aspectos éticos}

Primeiramente, o projeto foi encaminhado para o Comitê de Ética em Pesquisa do Hospital Psiquiátrico São Pedro. Após sua aprovação, foi iniciada a pesquisa, tendo o paciente assinado o termo de consentimento livre e esclarecido.

\section{Procedimento das sessões}

As sessões de TEE e TH foram individuais, com duração de 45 minutos e em número de seis, divididas ao 
longo de 2 semanas, sendo três na primeira semana e três na segunda. A distribuição das sessões e sua duração seguiram a orientação de estudos de efetividade envolvendo o TEE, que apregoam que o ideal é que sejam realizadas de seis a sete sessões com duração de 45 minutos a 1 hora $^{15}$. Antes da primeira sessão, foi aplicado o IATEGCRACK por um residente de psiquiatria treinado para tal.

\section{Montagem do protocolo individual}

A partir da análise do IATEGCRACK, foi montado um protocolo de exposições pela psicóloga, autora deste trabalho (R.B.A.), que trabalha na Unidade Jurandy Barcellos do Hospital Psiquiátrico São Pedro, a qual realizara treinamento com o Dr. Peter Monti em sua vinda ao Brasil, em 2009. O protocolo de exposição incluiu material similar ao que o paciente utilizava para consumir o crack e a simulação de situações de risco de recaída pontuadas como gerando mais fissura20.

Em cada sessão, era treinada alguma técnica de manejo de fissura: na primeira sessão, "espera passar"; na segunda, substituição por imagem positiva; na terceira, substituição por imagem negativa; na quarta, relaxamento respiratório; na quinta, beber ou comer algo alternativo; e na sexta, distração (comportamento alternativo). A utilização de uma mistura de técnicas (mix de técnicas) de escolha do paciente era estimulada nos 15 minutos finais da quinta e sexta sessões; nesta última, também eram devolvidas ao paciente as Ferramentas Pessoais de Manejo de Fissura pelo Crack. A escolha das técnicas seguiu o modelo preconizado por Monti et al. ${ }^{20}$, sendo acrescentado o relaxamento respiratório, que foi destacado por esses autores como técnica possível de ser utilizada ${ }^{20}$ e já avaliada por Zeni \& Araujo $^{26}$ para o manejo da fissura com dependentes de crack internados.

A ordem das técnicas seguiu, no estudo piloto do protocolo brasileiro, o protocolo original americano ${ }^{20}$ quanto às cinco primeiras sessões e quanto ao uso do mix de técnicas. No entanto, foi incluído no protocolo brasileiro, desde o início do estudo piloto, o relaxamento respiratório na quarta sessão, em vez da mera repetição das demais técnicas. O objetivo dessa inclusão foi aumentar o repertório de estratégias de enfrentamento para dependentes de crack, o que já havia sido destacado em outro estudo ${ }^{27}$. A ordem de apresentação da nova técnica foi atribuída por sorteio.

Após o estudo piloto, já na aplicação do TEE e do TH ao paciente descrito neste artigo, houve uma pequena modificação em relação ao protocolo original ${ }^{20}$, sendo a substituição por imagem positiva trocada de ordem com a substituição por imagem negativa. Essa troca ocorreu porque os pacientes do estudo piloto traziam, como técnica espontânea, desde a primeira sessão, a substituição por imagem positiva, porém a faziam de forma equivocada, o que poderia ser controlado se o treinamento fosse antecipado. Os pacientes imaginavam fatos positivos que já haviam ocorrido no passado, e não possíveis consequências futuras da sobriedade, como preconizado na literatura, tanto para esta técnica quanto para a substituição por imagem negativa ${ }^{20}$.

\section{Sessões}

Todas as sessões tiveram como terapeuta a psicóloga que montou o protocolo do paciente, acompanhada por um residente de psiquiatria que cronometrava e marcava os tempos e registrava a frequência cardíaca do paciente no Registro da Fissura e Confiança - Material do Terapeuta.

\section{Rapport}

Os princípios da habituação ${ }^{20}$ foram apresentados ao paciente, bem como a justificativa do TEE e do TH, explicando-se que a exposição contínua a esses gatilhos tende a diminuir seu potencial causador de fissura. Foram revistos os conceitos de fissura e confiança, e foi apresentado o Cartão de Fissura e Confiança, explicando-se como seria realizado o monitoramento durante as exposições. Foi explicado também o uso do cronômetro e do frequencímetro pelo terapeuta, e os registros que faria no Registro da Fissura e Confiança - Material do Terapeuta.

\section{Exposição in vivo}

O gatilho que o paciente mais pontuou no IATEGCRACK, a saber, a parafernália para uso da substância, era apresentado in vivo. Orientava-se que o paciente se permitisse sentir a fissura e que não usasse nenhuma ferramenta de enfrentamento. Solicitava-se que ele avisasse cada vez que o grau de fissura mudasse, dando uma nota para a mesma de 0 a 10 (de nenhuma a muita fissura), e dando uma nota também de 0 a 10 (de nenhuma a muita) para o grau de confiança que tinha de que não usaria o crack naquele momento. Pedia-se que o paciente avisasse quando considerasse que a fissura havia chegado ao nível mais alto.

O terapeuta mostrava, então, a parafernália, pedindo que o paciente tocasse/executasse o ritual de uso do crack (sem jamais imaginar-se usando a droga) e começava a cronometrar o tempo e a marcar a frequência cardíaca. Caso o nível de fissura chegasse a 10, ou baixasse espontaneamente, pedia-se para o paciente não induzir mais a fissura, apenas segurar a parafernália e usar a técnica para o manejo da fissura. 
O terapeuta tinha a função de monitorar as mudanças na fissura e na confiança, o tempo e a frequência cardíaca até a fissura baixar até 0 (ou, no mínimo, 2), quando, então, a exposição era interrompida. Nesse momento, discutia-se a experiência com a exposição, avaliava-se o uso espontâneo de ferramentas de enfrentamento e também a efetividade de cada uma das ferramentas utilizadas.

\section{Exposição imaginária}

A parafernália era deixada sobre a mesa, em um segundo plano, e iniciava-se a exposição ao gatilho pela imaginação que o paciente mais pontuou quanto à capacidade geradora de fissura no IATEGCRACK.

Solicitava-se que o indivíduo se imaginasse na situação geradora de fissura, até a fissura chegar ao ponto mais alto, mas sem se imaginar usando o crack. O terapeuta cronometrava o tempo, repetindo o procedimento da exposição in vivo. Depois, pedia-se que o paciente praticasse, usando a ferramenta trabalhada em cada sessão. As exposições eram feitas a todas as situações pontuadas no IATEGCRACK.

\section{Resultados}

\section{Avaliação para o TEE e o TH}

No IATEGCRACK, foi possível verificar que o sujeito usava o crack em um cachimbo de cano de PVC. Ele marcou como situações geradoras de fissura a exposição in vivo à pedra de crack (fissura $=10$ ) e ao cachimbo (fissura = 8), e, na imaginação, convites para usar crack (fissura $=7$ ), passar por locais de uso da droga (fissura $=6$ ), ter dinheiro (fissura $=6$ ) e brigar com a ex-companheira (fissura $=5$ ).

\section{Resultados obtidos em cada sessão}

A Tabela 1 apresenta o esquema de exposições e os resultados obtidos em cada sessão.

Na última sessão, foi aplicado o Questionário de Ferramentas Pessoais de Manejo de Fissura pelo Crack, e foram destacadas as seguintes imagens positivas: trabaIhar, imagens do filho e da família, ter uma vida normal, abraçar o filho. Como imagens negativas, foram listadas: lembrar da degradação de quem usa o crack, do estado físico com o crack, vergonha das pessoas, ver amigos com fissura e planejando roubo. Para o paciente, coisas que poderia comer ou beber durante a fissura foram: água, chocolates, balas, sucos, refrigerantes. As situações de risco identificadas foram: indiferença de familiares, medo de abandono pela família, quebrar regras, viajar com amigos, amigos convidarem para usar crack, frequentar locais de risco, sentir-se triste ou ansioso, autocrítica exagerada, ter dinheiro, sentir cheiro de crack, brigar com a ex-companheira, sair à noite sozinho, ver a droga.

\section{Avaliação 3 meses após a alta}

O paciente seguiu sendo acompanhado no Ambulatório de Dependência Química do Hospital Psiquiátrico São Pedro e no grupo Narcóticos Anônimos (NA), com frequência semanal. O tratamento ambulatorial foi realizado por um residente do $3^{\circ}$ ano de Psicologia supervisionado semanalmente pela psicóloga que realizou o TEE e o TH, que também trabalha nesse setor. $O$ tratamento seguiu $O$ modelo da terapia cognitivo-comportamental de Beck et al. $^{22}$, o qual aprofunda as crenças centrais do paciente e sua relação com as crenças aditivas, também avaliando situações de risco de recaída.

Foi realizado screening toxicológico para a detecção do uso de cocaína/crack e maconha após 3 meses da alta, com resultado negativo. O paciente relatava estar em abstinência de crack, maconha e álcool há 135 dias e de cocaína inalada há 140 dias. Estava com motivação equivalente a 10 (escala de 0 a 10) para continuar abstinente. Sua fissura, nos últimos 3 meses, teve valores de 0 para maconha, álcool e cocaína inalada e de 3 para crack (escala de 0 a 10).

As situações de risco de recaída que teve ao longo dos 3 meses após a alta foram: brigas com a ex-mulher, ter passado em determinados lugares relacionados ao uso do crack, ter lembranças ruins e ficar sozinho. As estratégias de enfrentamento que utilizou foram: relaxamento respiratório, substituição por imagem positiva, substituição por imagem negativa, comer algo alternativo, falar com alguém, buscar atendimento psicológico, ir ao grupo NA, distração e pedir que algum familiar ficasse junto dele. Ele considerou como mais efetivas a substituição por imagem positiva e a substituição por imagem negativa.

O paciente concluiu que o TEE e o TH o ajudaram na manutenção da abstinência do crack e das demais substâncias, pois o permitiram analisar fatores que não considerava como sendo de risco para recaída e aprendeu a lidar com eles, sempre tentando, como primeira opção, não se expor. Considerou que, nas situações em que acabou se expondo a risco, teve menos fissura por ter se acostumado a diversos estímulos associados ao uso do crack.

\section{Discussão}

O TEE combinado com TH demonstrou ser, no caso apresentado, um método coadjuvante ${ }^{20}$ que pode ser útil 
Tabela 1 - Detalhamento das sessões de tratamento de exposição de estímulos e treinamento de habilidades

\begin{tabular}{|c|c|c|c|c|c|c|}
\hline Exposição & $\begin{array}{l}\text { Fissura } \\
\text { inicial e } \\
\text { máxima }\end{array}$ & $\begin{array}{l}\text { Confiança } \\
\text { inicial e } \\
\text { máxima }\end{array}$ & $\begin{array}{c}\text { Tempo } \\
\text { até fissura } \\
10 \text { (ou } \\
\text { mais alta), } \\
\text { minutos }\end{array}$ & $\begin{array}{c}\text { Tempo } \\
\text { da fissura } 10 \\
\text { (ou mais alta) } \\
\text { a } 0, \text { minutos }\end{array}$ & $\begin{array}{c}\text { Frequência } \\
\text { cardíaca na } \\
\text { fissura } 10 \\
\text { (ou mais alta) } \\
\text { e na } 0\end{array}$ & Ferramenta (nota) \\
\hline \multicolumn{7}{|l|}{ Sessão 1} \\
\hline Parafernália & $9-10$ & $3-9$ & 12,08 & 10,27 & 104-99 & Nenhuma \\
\hline Parafernália & $7-10$ & $3-9$ & 5,43 & 8,42 & $98-100$ & Espera passar (8) \\
\hline \multicolumn{7}{|l|}{ Imaginação } \\
\hline Convite para usar crack & $10-10$ & $2-9$ & 3,44 & 6,86 & $98-97$ & Espera passar (8) \\
\hline \multicolumn{7}{|l|}{ Sessão 2} \\
\hline Parafernália & $7-10$ & 4-9 & 7,00 & 8,00 & $115-108$ & Espera passar (8) \\
\hline Parafernália & $5-10$ & $5-9$ & 7,00 & 6,00 & $109-108$ & Imagem positiva (10) \\
\hline \multicolumn{7}{|l|}{ Imaginação } \\
\hline Convite para usar crack & $5-10$ & $5-9$ & 7,12 & 6,05 & $109-108$ & Espera passar (8) \\
\hline Convite para usar crack & $5-10$ & $5-9$ & 4,00 & 7,00 & $109-108$ & Imagem positiva (10) \\
\hline \multicolumn{7}{|l|}{ Sessão 3} \\
\hline Parafernália & $5-10$ & $6-8$ & 6,70 & 2,97 & $99-100$ & Espera passar (8) \\
\hline Parafernália & $6-8$ & $7-9$ & 3,00 & 8,00 & $104-101$ & Imagem negativa ( 8 ) \\
\hline \multicolumn{7}{|l|}{ Imaginação } \\
\hline Convite para usar crack & $5-8$ & $7-9$ & 5,55 & 7,15 & $103-102$ & Espera passar (8) \\
\hline Convite para usar crack & $2-10$ & $8-9$ & 9,00 & 5,00 & $103-104$ & Imagem negativa (9) \\
\hline \multicolumn{7}{|l|}{ Sessão 4} \\
\hline Parafernália & $6-8$ & $7-9$ & 3,00 & 8,00 & $104-101$ & Espera passar (8) \\
\hline Parafernália & $4-8$ & $7-9$ & 4,92 & 3,14 & $104-108$ & $\begin{array}{l}\text { Relaxamento } \\
\text { Respiratório (8) }\end{array}$ \\
\hline \multicolumn{7}{|l|}{ Imaginação } \\
\hline Convite para usar crack & $4-8$ & $6-8$ & 4,05 & 3,33 & $104-107$ & Espera passar (8) \\
\hline Convite para usar crack & $4-9$ & $6-8$ & 4,04 & 4,01 & $107-107$ & $\begin{array}{l}\text { Relaxamento } \\
\text { Respiratório (9) }\end{array}$ \\
\hline Ver pessoas usando & $4-8$ & $7-9$ & 5,42 & 4,21 & $107-105$ & Espera passar (7) \\
\hline Ver pessoas usando & $4-9$ & $7-8$ & 3,12 & 2,67 & $109-104$ & $\begin{array}{l}\text { Relaxamento } \\
\text { Respiratório (9) }\end{array}$ \\
\hline \multicolumn{7}{|l|}{ Sessão 5} \\
\hline Parafernália & $4-8$ & $7-9$ & 3,25 & 2,66 & $107-106$ & Espera passar (8) \\
\hline Parafernália & $3-9$ & $7-9$ & 3,98 & 4,10 & $116-111$ & Comer bombom e beber água (10) \\
\hline \multicolumn{7}{|l|}{ Imaginação } \\
\hline Convite para usar crack & $3-6$ & $7-9$ & 2,32 & 1,53 & $117-115$ & Espera passar (8) \\
\hline Convite para usar crack & $3-8$ & $8-9$ & 3,02 & 3,18 & $120-120$ & Comer bombom e beber água (10) \\
\hline Ver pessoas usando & $3-8$ & $8-9$ & 3,06 & 2,48 & $120-119$ & Espera passar (8) \\
\hline Ver pessoas usando & $4-10$ & $7-9$ & 2,84 & 2,40 & $120-118$ & Comer bombom e beber água (10) \\
\hline Ter dinheiro & $4-9$ & $6-9$ & 3,40 & 3,47 & $119-116$ & Espera passar (8) \\
\hline Ter dinheiro & $4-9$ & $7-9$ & 3,20 & 2,47 & $118-116$ & Mix de técnicas (10) \\
\hline \multicolumn{7}{|l|}{ Sessão 6} \\
\hline Parafernália & $3-8$ & $8-9$ & 3,11 & 2,40 & $104-108$ & Espera passar (9) \\
\hline Parafernália & $3-8$ & $8-9$ & 4,22 & 2,34 & $109-104$ & Distração (9) \\
\hline \multicolumn{7}{|l|}{ Imaginação } \\
\hline Convite para usar crack & $3-7$ & $8-9$ & 5,21 & 2,11 & $108-106$ & Espera passar (9) \\
\hline Convite para usar crack & $3-7$ & $8-9$ & 6,00 & 2,05 & $108-107$ & Distração (9) \\
\hline Ver pessoas usando & $4-8$ & $8-9$ & 5,57 & 2,28 & $107-107$ & Espera passar (9) \\
\hline Ver pessoas usando & $4-8$ & $8-10$ & 4,26 & 2,08 & $107-108$ & Mix de técnicas (10) \\
\hline Ter dinheiro & $4-8$ & $8-10$ & 4,00 & 2,11 & $106-107$ & Espera passar (8) \\
\hline Ter dinheiro & $4-8$ & $8-10$ & 5,04 & 2,06 & $106-105$ & Mix de técnicas (10) \\
\hline Briga com ex-companheira & $4-8$ & $8-10$ & 5,22 & 2,43 & $106-105$ & Espera passar (8) \\
\hline Briga com ex-companheira & $4-8$ & $8-10$ & 5,55 & 2,21 & $106-105$ & Mix de técnicas (10) \\
\hline
\end{tabular}


no tratamento de dependentes de $\mathrm{crack}^{19}$. No entanto, devemos salientar que este relato é de um único caso, e portanto não podemos generalizar os achados para todos os pacientes com essa patologia. O paciente conseguiu permanecer em abstinência por 3 meses após a intervenção, mas também passou por uma série de outros tratamentos, como entrevista motivacional ${ }^{21}$, prevenção de recaída ${ }^{23}$ e a terapia cognitivo-comportamental de Beck et al.22, o que pode confundir os resultados. Assim, o fato de o paciente ter atribuído ao TEE e ao TH a menor intensidade de sua fissura pós-alta também passa por um entendimento subjetivo.

No decorrer das exposições, pôde-se observar que a fissura e a confiança (autoeficácia) variavam juntas e de forma inversa, o que já havia sido observado por Araujo et al. ${ }^{27}$. Isso demonstra que o $\mathrm{TH}$ de enfrentamento à medida em que aumenta a autoeficácia pode auxiliar no manejo da fissura 27.

O fenômeno da habituação ${ }^{20}$ pôde ser observado durante as exposições tanto por meio do tempo necessário para diminuir a fissura nas últimas sessões (2 segundos, aproximadamente), quanto pelo valor mais alto da fissura, que no início chegava a 10 e, ao final, não ultrapassou 8 . A confiança, na última consulta, já iniciava mais alta (nota 8 , contra nota 3 na primeira sessão).

O paciente avaliado apresentou demora para que sua fissura chegasse ao ponto mais alto e mais demora ainda para que a fissura passasse, sendo isso mais evidente, como já citado, nas primeiras sessões. Segundo Monti et al. $^{20}$, os pacientes alcoolistas dificilmente levam mais de 8 minutos esperando passar sua fissura, e esse valor foi ultrapassado ou atingido em mais de um momento em nossa exposição. Esse dado é importante, pois torna a exposição a cada estímulo mais longa e faz com que talvez um protocolo com maior duração tenha que ser planejado para dependentes de crack. Não houve essa necessidade em nosso estudo, mas fica o dado como um alerta para novas pesquisas sobre o tema. Além disso, devemos refletir que esse paciente apresentava características de autocontrole e um alto nível de exigência, o que pode ter interferido nesses achados.

Um dado que chamou atenção foi a pouca variação na frequência cardíaca do paciente ao serem apresentados estímulos evocadores de fissura ou mesmo após ser identificada, através do autorregistro, uma diminuição na fissura. Esse achado contradiz os de vários autores $^{13,14}$ que associam alterações na frequência cardíaca com a apresentação de estímulos e com a variação da fissura, mas está de acordo com o que verificaram Ahmed \& Koob ${ }^{18}$ e Childress et al. ${ }^{19}$ quanto à dificuldade de simular, no contexto do tratamento, os estímulos interoceptivos associados ao uso da droga. Como este artigo descreve um único caso, não podemos discutir se o fenômeno é característico do dependente de crack ou se foi alguma peculiaridade do nosso sujeito de pesquisa.

As estratégias de enfrentamento da fissura treinadas no protocolo foram consideradas efetivas pelo paciente (notas de 7 a 10), e o uso de mais de uma técnica demonstrou ser muito efetivo (nota 10 e pouco tempo necessário para baixar a fissura), o que se assemelha ao preconizado por Monti et al. ${ }^{20}$. De todas as estratégias, ele considerou como as mais efetivas após a alta a substituição por imagem positiva e a substituição por imagem negativa, dado corroborado por alguns outros autores $^{10,15,20}$.

Após 3 meses da alta hospitalar, o paciente continuava com fissura nota 3 pelo crack. Isso pode ocorrer, conforme já alertado na literatura ${ }^{19}$, porque não há como o indivíduo, durante o TEE, ser exposto a todos os gatilhos que encontrará ao retomar sua rotina de vida.

O paciente destacou que o TEE e o TH possibilitaram que ele identificasse fatores de risco de recaída e aprendesse a lidar com eles, destacando a importância de não se expor a eles. Isso é muito importante, pois o objetivo da técnica não é estimular os pacientes a se confrontarem com as situações de risco, mas treinar habilidades caso esse encontro seja inevitável. As estratégias de coping de confronto não devem ser estimuladas, já que aumentam o risco de recaída ${ }^{20,27}$. O paciente também avaliou que, nas situações de risco a que se expôs pós-alta, teve menos fissura, o que associou ao fato de ter se acostumado a diversos estímulos durante o TEE e o $\mathrm{TH}^{19,20}$. Quando questionado sobre se poderia haver alguma outra explicação para esse fato, disse não conseguir pensar em nenhum outro motivo.

O presente estudo lança a hipótese de que talvez o TEE associado ao TH para o manejo da fissura possa ser útil como coadjuvante no tratamento de dependentes de crack. Seu uso deve ser avaliado em ensaios clínicos para demonstrar seu real benefício.

\section{Referências}

1. Guimarães CF, Santos DVV, Freitas RC, Araujo RB. Perfil do usuário de crack e fatores relacionados a criminalidade em unidade de internação para desintoxicação no Hospital Psiquiátrico São Pedro de Porto Alegre (RS). Rev Psiquiatr Rio Gd Sul. 2008;30(2):101-8.

2. Seibel SD, Toscano Jr A. Dependência de drogas. São Paulo: Atheneu; 2001.

3. Reid MS, Thakkar V. Valproate treatment and cocaine cue reactivity in cocaine dependent individuals. Drug Alcohol Depend. 2009;102(1-3):144-50.

4. Silva de Lima M, Farrell M, Lima Reisser AA, Soares $B$. Withdrawn: antidepressants for cocaine dependence. Cochrane Database Syst Rev. 2010(2):CD002950. 
5. Minozzi S, Amato L, Davoli M, Farrell M, Lima Reisser AARL, Pani PP, et al. Anticonvulsants for cocaine dependence. Cochrane Database Syst Rev. 2008(2):CD006754.

6. Kampman KM, Pettinati H, Lynch KG, Dackis C, Sparkman $\mathrm{T}$, Weigley $\mathrm{C}$, et al. A pilot trial of topiramate for the treatment of cocaine dependence. Drug Alcohol Depend. 2004;75(3):233-40.

7. Karila L, Reynaud M. [Therapeutic approaches to cocaine addiction]. Rev Prat. 2009;59(6):830-4.

8. Montoya ID, Vocci F. Novel medications to treat addictive disorders. Curr Psychiatry Rep. 2008;10(5):392-8.

9. Barth KS, Malcolm RJ. Disulfiram: an old therapeutic with new applications. CNS Neurol Disord Drug Targets. 2010;9(1):5-12.

10. Araujo RB, Oliveira MS, Pedroso RS, Miguel AC, Castro MGT. Craving e dependência química: conceito, avaliação e tratamento. J Bras Psiquiatr. 2008;57(1):57-63.

11. Taylor JR, Olausson P, Quinn JJ, Torregrossa MM. Targeting extinction and reconsolidation mechanisms to combat the impact of drug cues on addiction. Neuropharmacology. 2009;56(1):186-95.

12. Volkow ND, Wang GJ, Telang F, Fowler JS, Logan J, Childress $A R$, et al. Cocaine cues and dopamine in dorsal striatum: mechanism of craving in cocaine addiction. J Neurosci. 2006;26:658-88.

13. Foltin RW, Haney M. Conditioned effects of environmental stimuli paired with smoked cocaine in humans. Psychopharmacology. 2000;149:24-33.

14. Field M, Duka T. Cues paired with a low dose of alcohol acquire conditioned incentive properties in social drinkers. Psychopharmacology. 2002;159:325-34.

15. Monti PM, Rohsenow DJ. Coping-skills training and cueexposure therapy in the treatment of alcoholism. Alcohol Res Health. 1999;23(2):107-15.

16. Aragon MBQ, Labrador FJ, Arce F. Evaluation of a group cue-exposure treatment for opiate addicts. Span J Psychol. $2005 ; 8(2): 229-37$.

17. Lee JH, Ku J, Kim K, Kim B, Kim IY, Yang BH, et al. Experimental application of virtual reality for nicotine craving through cue exposure. Cyberpsychol Behav. 2003;6(3):275-80.
18. Ahmed SH, Koob GF. Rapid extinction of cocaine craving: toward a novel cue exposure therapy. Nature. http://dx.doi. org/10.1038/npre.2009.2980.1. Acessado set 2010.

19. Childress AR, Ehrman R, Rohsenow DJ, Robbins SJ, OBrien CP. Classically conditioned factors in drug dependence. In: Lowinson JH, Ruiz P, Millman RB, Langrod JG, eds. Substance abuse: a comprehensive textbook. 2nd ed. Baltimore: Williams and Wilkins; 1992. p. 56-69.

20. Monti PM, Kadden RM, Rohsenow DJ, Cooney NL, Abrams DB. Tratando a dependência de álcool: um guia de treinamentos das habilidades de enfrentamento. São Paulo: Roca; 2005.

21. Miller WR, Rollnick S. Entrevista motivacional: preparando as pessoas para a mudança de comportamentos adictivos. Porto Alegre: Artmed; 2001.

22. Beck A, Newman C, Wright F. Terapia cognitiva de las drogodependencias. Barcelona: Paidos Ibérica; 2000.

23. Marlatt A, Gordon J. Prevenção de recaída: estratégias de manutenção no tratamento de comportamentos adictivos. Porto Alegre: Artes Médicas; 1993.

24. Organização Mundial da Saúde. Classificação de transtornos mentais e de comportamento da CID-10: descrições clínicas e diretrizes diagnósticas. Porto Alegre: Artes Médicas; 1993.

25. Columbia University, Biometrics Research Department. Structured Clinical Interview for DSM Disorders (SCID). http:// cpmcnet.columbia.edu/dept/scid/. Acessado 10 abr 2010.

26. Zeni TC, Araujo RB. O relaxamento respiratório no manejo do craving e dos sintomas de ansiedade em dependentes de crack. Rev Psiquiatr Rio Gd Sul. 2009;31(2):116-9.

27. Araujo RB, Pansard M, Boeira BU, Rocha NS. As estratégias de coping para o manejo da fissura de dependentes de crack. Rev HCPA. 2010;30(1):36-42.

\section{Correspondência}

Renata Brasil Araujo

Cognitá - Clínica de Terapia Conitivo-Comportamental

Rua Mostardeiro, 5/605, Bairro Moinhos de Vento

90430-001 - Porto Alegre, RS - Brasil

Tel.: (51) 3019.3560

E-mail: renataudbrasil@terra.com.br 